\title{
Effect of Radix Hedysari Polysaccharide on Glioma by Cell Cycle Arrest and TNF- $\alpha$ Signaling Pathway Regulation
}

\author{
Xiaolong Du, ${ }^{1,2}$ Yujing Zhao, ${ }^{2}$ Yongqian $\mathrm{Ma}^{2}{ }^{2}$ Hongshun Xing, ${ }^{2}$ and Xingang $\mathrm{Li}^{\mathrm{D}}{ }^{1}$ \\ ${ }^{1}$ Qilu Hospital of Shandong University, China \\ ${ }^{2}$ Weifang People's Hospital, China \\ Correspondence should be addressed to Xingang Li; lixg@sdu.edu.cn
}

Received 10 March 2019; Revised 6 June 2019; Accepted 4 July 2019; Published 25 July 2019

Guest Editor: Jingxiao Chen

Copyright (C) 2019 Xiaolong Du et al. This is an open access article distributed under the Creative Commons Attribution License, which permits unrestricted use, distribution, and reproduction in any medium, provided the original work is properly cited.

\begin{abstract}
Objective. To investigate the possible development of radix hedysari polysaccharide as an antiglioma drug, we studied the effect of radix hedysari polysaccharide on glioma cells in vitro and the growth of glioma in nude mice and on the phagocytosis of macrophages in nude mice with glioma. Methods. The effect of radix hedysari polysaccharide on the growth of glioma was studied based on U251 cell line in vitro. The effect of radix hedysari polysaccharide on the growth of glioma was studied in vivo. The growth inhibition rate of radix hedysari polysaccharide on U251 cell line was determined by the MTT assay. The cell cycle of U251 was analyzed by flow cytometry. The expression of cytokines in U251 cells and tumor tissues was detected using PCR. The phagocytosis of macrophages in the serum of glioma nude mice was detected by Giemsa staining. TNF- $\alpha$ signaling pathway proteins in the serum of glioma nude mice were detected by ELISA. Results. Radix hedysari polysaccharide inhibited the growth of U251 cells, induced apoptosis in G1 phase by cell cycle arrest, and facilitated apoptosis in glioma mice by regulating cell cycle. Mice injected with radix hedysari polysaccharide showed delayed tumor growth and grew slowly. Radix hedysari polysaccharide enhanced the phagocytosis of macrophages in glioma nude mice. Radix hedysari polysaccharides could inhibit tumor development by regulating the immune function of tumor mice and affecting the TNF- $\alpha$ signaling pathway. Conclusion. Radix hedysari polysaccharide can effectively inhibit the growth of glioma and affect the TNF- $\alpha$ signaling pathway, thus playing an antiglioma role.
\end{abstract}

\section{Introduction}

Glioma is the most common and fatal primary central nervous system tumor in adults. Although the number of the therapeutic drugs for glioma is increasing, the therapeutic effect on glioma is still unsatisfactory. It was investigated that the survival time after a combined approach of surgical resection followed by chemoradiotherapy is short and the prognosis of patients with glioma is extremely poor [1]. Therefore, it is still urgent to seek for the excellent drug for the treatment of glioma. The primary task of developing new drugs is to explore the effect of drugs on proliferation and invasion of glioma and molecular mechanisms involved. Polysaccharides that widely exist in many plants are macromolecular substances with various biological activities, which have not only attracted extensive attention as excellent biological packaging materials but have also been widely used in enhancing immu- nity, anticancer, and anti-inflammation [2-5]. It was reported that polysaccharides induced tumor cell cycle arrest by regulating cyclin $\mathrm{E}$ and $\mathrm{D}$ [3] or facilitated apoptosis in glioma cells by altering the Bax/Bcl-2-p53 axis [6]. Polysaccharides extracted from radix hedysari have attracted much attention due to their antitumor activity, immunomodulatory effects, and antioxidant properties [7], but there are few reports on the effects of radix hedysari polysaccharide on glioma.

In the occurrence and development of glioma, macrophages have two different characters, which perform functions of promoting growth and invasion of tumor or killing tumor cells in different microenvironments [8]. And it was reported that radix hedysari polysaccharide can significantly improve the phagocytosis of macrophages [9]. Tumor necrosis factor (TNF- $\alpha$ ) is a key cytokine involved in inflammation, immunity, cellular homeostasis, 
and tumor progression. TNF- $\alpha$ plays an essential role on the normal cell proliferation and function of macrophages, NK cells, T cells, B cells, and dendritic cells, and it is an important effector molecule for cell-mediated killing of some tumors [10]. Therefore, we proposed that radix hedysari polysaccharides can affect tumor growth and immune response through TNF- $\alpha$ signaling pathway. To explore the effect of radix hedysari polysaccharide on glioma, we studied the effects of radix hedysari polysaccharide on glioma cells in vitro and the growth of tumors in nude mice with glioma and on the phagocytosis of macrophages in nude mice with glioma.

\section{Materials and Methods}

The preparation of radix hedysari polysaccharide, referring to the preparation method of RHPs-4 by Dongfeng Wei, is as follows.

2.1. Cell Culture. In our studies, U251 cells were provided by the Cell Bank of Shanghai Institutes for Biological Sciences, CAS. U251 cells were cultured in DMEM containing 10\% fetal bovine serum and $100 \mathrm{U} / \mathrm{ml}$ penicillin-streptomycin and were kept in a $37^{\circ} \mathrm{C}$ incubator with $5 \% \mathrm{CO}_{2}$.

\subsection{Establishment of Glioma Model of Nude Mice. Twenty} immunosuppressed nude mice (BALB/c-nu; 4-6 weeks old; $16-18 \mathrm{~g}$ ) were provided by the Beijing Vital River Laboratory Animal Technology Co. Ltd. and randomly divided into 2 groups. Mice were kept in a pathogen-free environment. All animal experiments conformed to the international standards for the care and treatment of experimental animals. U251 cells $\left(5^{*} 105\right)$ in logarithmic growth phase were taken for preparing cell suspension. The cell suspension was injected subcutaneously into the right tissue of nude mice to establish a xenograft model. Xenografted U251 glioma model of nude mice was randomly divided into the blank group and the RHPS group after inoculation. The mice were administered subcutaneously in the nape of the neck once a day for 24 consecutive days. The size of the xenografted tumor was measured with a caliper every 4 days during the 24-day observation period. The volume of the tumor was calculated according to the formula of volume $=L^{*} S^{2} / 2$, and the growth curve of the tumor was drawn, where $L$ is the longest tumor diameter and $S$ is the shortest diameter of the twodimensional tumor. The animals were sacrificed after observation, and the tumor weight was measured. Xenografted tumor tissue was taken and then prepared for cell suspension using a tissue homogenizer.

2.3. In Vitro Growth Inhibition Assay. The inhibitory effect of RHPS on human glioma cell line U251 or glioma in nude mice was studied by the MTT assay. Tumor cell suspension was prepared with cells $(8 \times 103$ cells/well $)$ seeded in $96-$ well plates. The different concentrations of RHPS or CPT were added, and cells were cultured for 48 hours at $37^{\circ} \mathrm{C}$. Then, the culture medium was removed, and $0.5 \mathrm{mg} / \mathrm{ml}$ of MTT reagent $20 \mu \mathrm{l}$ was added for incubation for 4 hours. The MTT reagent was discarded, and DMSO (150 $\mu \mathrm{l} /$ well $)$

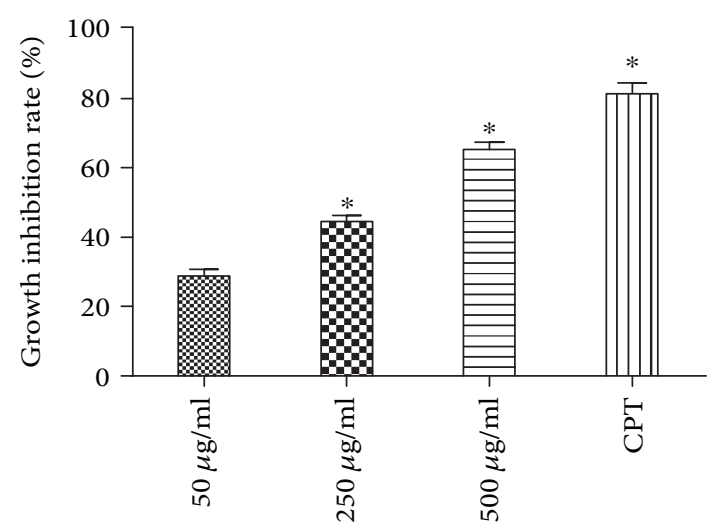

FIGURE 1: The growth inhibition of different concentrations of radix hedysari polysaccharide on U251 cells detected by the MTT assay. ${ }^{*} P<0.05$, comparing the $50 \mu \mathrm{g} / \mathrm{ml}$ RHPS experimental groups.

was added. The absorbance at $570 \mathrm{~nm}$ was measured by a spectrophotometer.

2.4. Cell Cycle Analysis. The effect of RHPS on cell cycle of U251 was detected using flow cytometry. U251 glioma cells $\left(4 \times 10^{5}\right)$ were incubated with RHPS $(500 \mu \mathrm{g} / \mathrm{ml})$ for 48 hours and then rinsed with PBS twice. Afterwards, the cells were fixed using $75 \%$ ethanol at $4^{\circ} \mathrm{C}$ overnight. Before flow cytometry analysis, the fixed cells were washed with PBS twice and stained with $50 \mu \mathrm{g} / \mathrm{ml}$ PI solution for $30 \mathrm{~min}$ at room temperature. The stained cells were transferred and flowed through a test tube with a $40 \mu \mathrm{m}$ nylon mesh strainer for flow cytometry analysis.

2.5. Detection of Cytokine Expression. Expression levels of Bax, Bcl-2, caspase-3, and P53 mRNA were detected by RTPCR. Sample cells or an appropriate amount of tumor tissues was collected, ground, and homogenized for subsequent RNA extraction. RNA extraction was carried out in accordance with the manufacturer's instructions. RNA concentration was quantitated by value of O.D before reverse transcription experiment, and the product cDNA was stored at $-20^{\circ} \mathrm{C}$. The PCR reactions were performed according to the instructions of the fluorescent quantitative PCR kit in which cDNA served as a template. Quantitative analysis was performed using $2-\Delta \Delta \mathrm{ct}$.

2.6. Western Blot Assay. We adopted western blot to detect the protein expression levels of cytokines Bax (Abcam, ab32503), Bcl-2 (Abcam, ab32124), caspase-3 (Abcam, ab13847), and p53 (Abcam, ab26). GAPDH (Abcam, ab181602) was used as an internal reference gene, and antibodies were used according to the product insert for Bax, Bcl-2, caspase-3, p53, E-cadherin, and GAPDH. Scanning imaging was performed using an imaging system (TANON, Tanon-5200), and the desired result has been achieved by resensing or shortening the photosensitizing time depending on the intensity of the band.

2.6.1. Secondary Antibody. Membranes incubated with primary antibody were washed three times with TBST for 


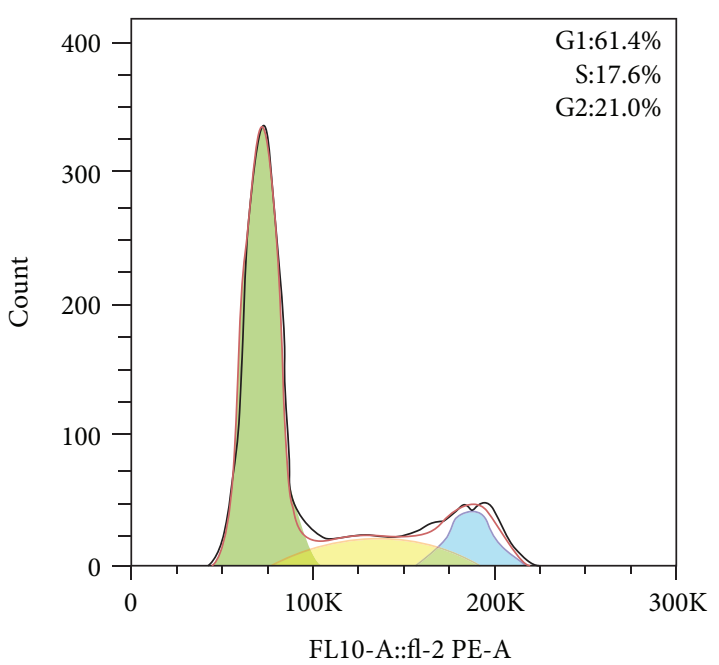

(a)

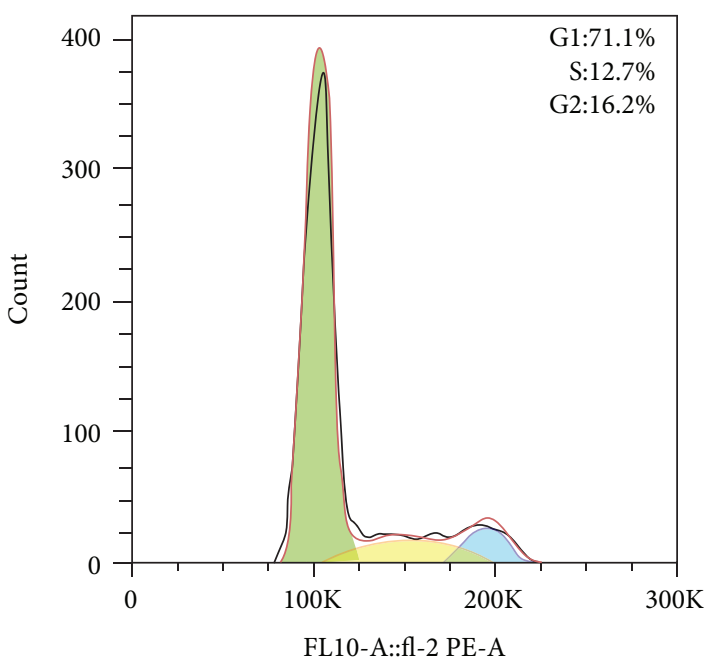

(b)

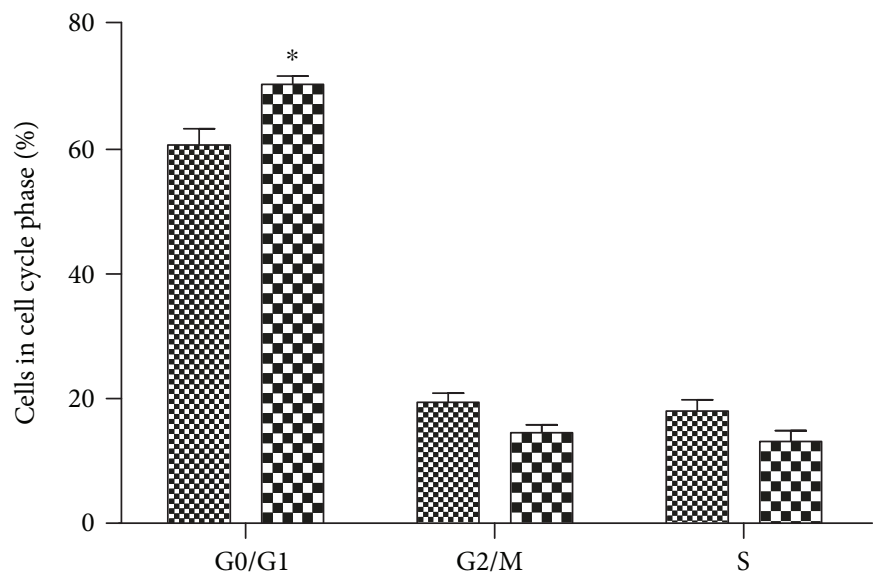

\% Blank

$500 \mu \mathrm{g} / \mathrm{ml}$ RHPs

(c)

Figure 2: G1 arrest in glioma cells induced by radix hedysari polysaccharide. ${ }^{*} P<0.05$, comparing the blank experimental groups.

5 min. The HRP-conjugated secondary antibody was subsequently diluted $1: 1000$ according to the amount and incubated with the membrane for $1 \mathrm{~h}$ at $37^{\circ} \mathrm{C}$. They were washed three times with TBST for $5 \mathrm{~min}$.

2.7. Macrophage Activity. Macrophage activity was detected by macrophage phagocytosis of chicken erythrocytes. Twenty-four hours after the last administration, the mice were intraperitoneally injected with $5 \%$ chicken red blood cell suspension $1 \mathrm{ml}$ and sacrificed 1.5 hours later. The smear of rat peritoneal fluid was incubated at constant temperature of $37^{\circ} \mathrm{C}$ for $30 \mathrm{~min}$, washed and dried, and then stained with Giemsa-Reiter solution for $15 \mathrm{~min}$. Afterwards, it was washed and dried again with distilled water and observed under an oil immersion lens for counting cells. The phagocytic rate (number of macrophages that phagocytose chicken red blood cells/300 macrophages multiplied by 100\%) and phagocytic index (total number of phagocytized chicken erythrocytes/300 macrophages) were calculated.
2.8. Detection of TNF- $\alpha$ Signaling Pathway. We further detected TNF- $\alpha$, NF-KB P56, and caspase- 3 in the serum of glioma nude mice by ELISA.

\section{Results}

3.1. Growth Inhibition Test of Radix Hedysari Polysaccharide on Glioma Cell Lines. The study set three groups: the blank control group, the RHPS experimental group $(50 \mu \mathrm{g} / \mathrm{ml}$, $250 \mu \mathrm{g} / \mathrm{ml}$, and $500 \mu \mathrm{g} / \mathrm{ml}$ ), and the camptothecin- (CPT-) positive control group. The growth inhibition rate of radix hedysari polysaccharide on glioma cell lines was determined by the MTT assay. As shown in Figure 1, the growth inhibition rate of radix hedysari polysaccharide on glioma cell lines showed dose dependency. The inhibition rate in the low-dose group was $29.5 \%$, and the rate in the middle-dose group was $45.3 \%$. The inhibition rate in the high-dose group was $66.0 \%$, revealing that the growth of cells in the group was significantly suppressed compared with that of other groups 


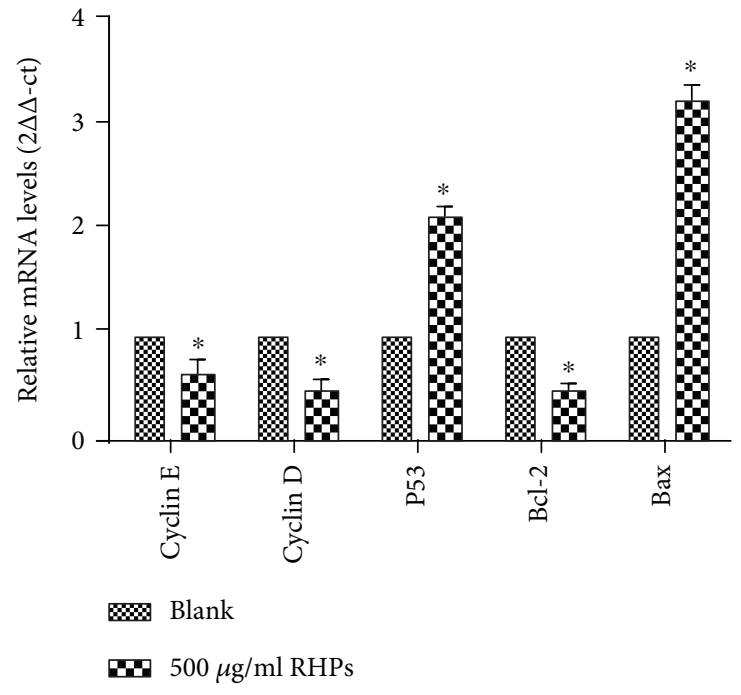

(a)

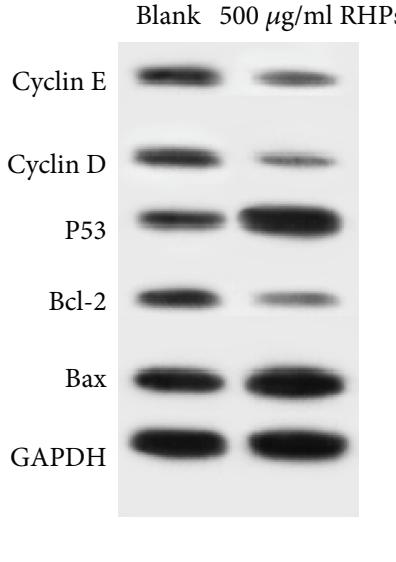

(b)

FIGURE 3: Effect of radix hedysari polysaccharide on cytokines in glioma cell lines. ${ }^{*} P<0.05$, comparing the blank experimental groups.

(Figure 1). These results indicated that radix hedysari polysaccharide obviously suppressed the growth of U251 cells and $500 \mu \mathrm{g} / \mathrm{ml}$ of radix hedysari polysaccharide had a better effect of inhibiting cell growth.

\subsection{Effect of Radix Hedysari Polysaccharide on Cell Cycle of} Glioma Cell Lines. In order to further explore the inhibitory effect of radix hedysari polysaccharide on U251 cells, we studied the changes in the cell cycle of U251 cells and analyzed their cell cycle distribution by flow cytometry. Figure 2 shows the effects of radix hedysari polysaccharide on the cell cycle of U251 cells (G1, S, and G2). As compared with the control group (the cell ratio in the G1 phase was $61.4 \%$ ), the ratio of U251 cells in the G1 phase was significantly increased in the experimental group treated with $500 \mu \mathrm{g} / \mathrm{ml}$ of radix hedysari polysaccharide (the cell ratio in the $\mathrm{G} 1$ phase was $71.1 \%, P<0.05)$. The results indicated that radix hedysari polysaccharide can suppress the growth of U251 cells and induce apoptosis in the G1 phase by cell cycle arrest (Figure 2).

3.3. Effect of Radix Hedysari Polysaccharide on Cytokines in Glioma Cell Lines. The cytokine expressions of the control group and the administration group treated with $500 \mu \mathrm{g} / \mathrm{ml}$ radix hedysari polysaccharide were detected by PCR. The results showed that the expression levels of P53 and Bax mRNA were significantly increased $(P<0.05)$, while the expression level of Bcl-2 mRNA was significantly decreased $(P<0.05)$, which indicated that radix hedysari polysaccharide induced the apoptosis of U251 cells by changing the expression of cytokine genes (see Figure 3). As compared with the control group, the expression levels of cyclin $\mathrm{E}$ and cyclin $\mathrm{D}$ in the administration group were significantly decreased $(P<0.05)$ (see Figure 3(a)) and the results of the western blot assay were consistent with those of the PCR assay (see Figure $3(\mathrm{~b})$ ), which further indicated that radix

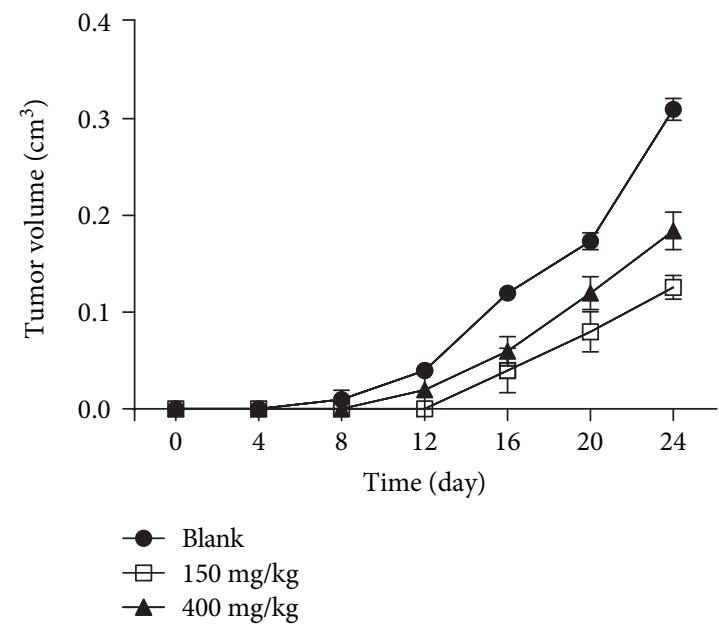

FIGURE 4: Effect of radix hedysari polysaccharide on the volume of tumor in nude mice with glioma.

hedysari polysaccharide induced apoptosis in the G1 phase through cell cycle arrest.

3.4. Effect of Radix Hedysari Polysaccharide on Mean Mass and Volume of Tumors in Nude Mice with Glioma. We have demonstrated that radix hedysari polysaccharide inhibited the proliferation of U251 cells in vitro by inducing G1 cell cycle arrest. The glioma model of nude mice was established to study the effect of radix hedysari polysaccharide on the growth of glioma in vivo. As shown in Figure 4, the mice injected with radix hedysari polysaccharide grew slowly and the progression of tumors in vivo was delayed. As compared with the high-dose group $(400 \mathrm{mg} / \mathrm{kg}$ ), the growth delay of the low-dose group $(150 \mathrm{mg} / \mathrm{kg}$ ) was significantly increased and the volume of tumor after 24 days of administration was significantly decreased $(P<0.05)$. After 24 days of 


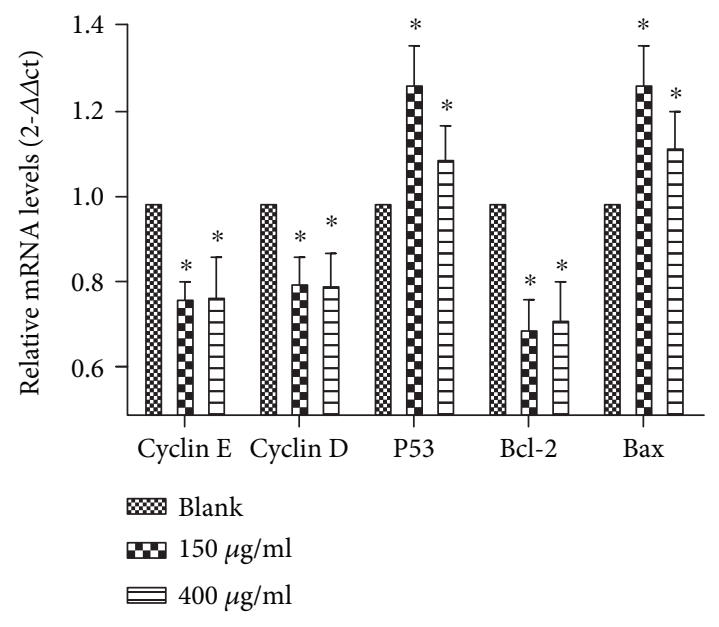

FIGURE 5: Effect of radix hedysari polysaccharide on cytokines in glioma tissues.

administration, the masses of the blank control group, the low-dose group, and the high-dose group were $0.32 \pm 0.17 \mathrm{~g}$ , $0.13 \pm 0.08 \mathrm{~g}$, and $0.15 \pm 0.05 \mathrm{~g}$, respectively. The tumor mass of mice injected with radix hedysari polysaccharide was decreased significantly $(P<0.05)$, and the tumor mass of the low-dose group was decreased significantly $(P<0.05)$ compared with that of the high-dose group after 24 days of administration. These data suggested that radix hedysari polysaccharide can obviously inhibit tumorigenesis in nude mice.

3.5. Effect of Radix Hedysari Polysaccharide on Cytokines in Glioma Tissues. We examined the effect of radix hedysari polysaccharide on the changes in cytokine expression of tumor tissue cells by PCR. The results showed that the expression levels of P53 and Bax mRNA were significantly increased $(P<0.05)$, which was similar with the results in vitro, while the expression of Bcl-2 mRNA was significantly decreased $(P<0.05)$. The results indicated that the radix hedysari polysaccharide induced the apoptosis of tumor cells by changing the expression of cytokine genes in vivo (see Figure 5). As compared with the control group, the levels of cyclin $\mathrm{E}$ and cyclin $\mathrm{D}$ in tumor tissues of the administration group were significantly reduced $(P<0.05)$, indicating that the radix hedysari polysaccharide induced the apoptosis in mice with glioma by regulating the cell cycle (see Figure 5). The effect of radix hedysari polysaccharide on cytokine expression of tumor tissue cells was not dose dependent.

3.6. Effect of Radix Hedysari Polysaccharide on Phagocytosis of Macrophages in the Serum of Nude Mice with Glioma. Differentiation and development of glioma are closely related to the phagocytosis of macrophages. We detected the phagocytosis of macrophages in the serum of nude mice with glioma by Giemsa staining. The results showed that the phagocytic rate and index of the nude mice with glioma were significantly lower than those of the normal nude mice $(P<0.05)$, while the phagocytic rate and index of nude mice with glioma significantly increased after administration of radix hedysari polysaccharide $(P<0.05)$. There were also remarkable differences between the high-dose group and the low-dose group (see Table 1). The results showed that radix hedysari polysaccharide enhanced the phagocytosis of macrophages in the nude mice with glioma.

3.7. Effect of Radix Hedysari Polysaccharide on TNF- $\alpha$ Signaling Pathway in Nude Mice with Glioma. As radix hedysari polysaccharide can enhance the phagocytosis of macrophages, we further detected such TNF- $\alpha$ signaling pathway proteins as TNF- $\alpha$, NF-KB P56, and caspase- 3 that are closely related to the macrophages in the serum of the nude mice with glioma by ELISA. The results showed that the expressions of TNF- $\alpha$ and caspase- 3 in the nude mice with glioma were significantly lower than those in normal nude mice $(P<0.05)$, while the expression of NF-KB P56 was significantly overexpressed $(P<0.05)$. And this was obviously improved after the administration of radix hedysari polysaccharide. As compared with the nude mice with glioma, the expressions of TNF- $\alpha$ and caspase- 3 in the serum were significantly elevated in the administration group $(P<0.05)$, and the highly expressed NF-KB P56 was significantly inhibited $(P<0.05)$ (see Table 2).

\section{Discussion}

Polysaccharides play an antitumor role by affecting tumor differentiation and apoptosis, changing intracellular signal transmission and immune regulation [11, 12]. This study found that radix hedysari polysaccharide inhibited the growth of U251 in vitro and arrested cells in the G1 phase, which is consistent with the results reported in other studies [13]. At present, there are many apoptosis-induced drugs, but their toxic and side effects are also serious [14]. As a macromolecular drug extracted from plants, radix hedysari polysaccharide has better effects in enhancing immunity, anticancer, and anti-inflammation and has less toxic and side effects, which therefore is a good candidate drug.

Studies have found that macrophages play an active role in the growth and invasion of glioma. However, as the main cells involved in the immune system, they can secrete TNF to induce apoptosis of tumor cells or directly engulf tumor cells under variable microenvironment [15]. The study found that phagocytosis of macrophages was significantly enhanced by the stimulation of radix hedysari polysaccharide. The link between inflammation and cancer has been well realized in the treatment of many cancers, and inflammation is considered the seventh characteristic of cancer. TNF- $\alpha$ is the main mediator of cancer-associated inflammation and is a factor that promotes tumor growth. The proinflammatory effect of TNF- $\alpha$ was mainly due to its ability to activate NF- $\kappa \mathrm{B}$, while its antitumor effect was due to the activation of caspase- 3 and the induction of apoptosis. When exposed to TNF- $\alpha$, NF- $\kappa$ B is activated and results in the expression of a variety of inflammation-related genes in almost every type of cell. However, the sustained activation of NF- $\kappa \mathrm{B}$ is associated with several aspects of tumorigenesis, such as promoting tumor cell proliferation, preventing apoptosis of drug- 
TABLE 1: Enhancement of phagocytosis of macrophages in nude mice with glioma by radix hedysari polysaccharide.

\begin{tabular}{lcccc}
\hline Group & $\begin{array}{c}\text { Normal nude mice } \\
(n=6)\end{array}$ & $\begin{array}{c}\text { Nude mice with glioma } \\
(n=6)\end{array}$ & $\begin{array}{c}150 \mu \mathrm{g} / \mathrm{ml} \text { dose group } \\
(n=6)\end{array}$ & $\begin{array}{c}400 \mu \mathrm{g} / \mathrm{ml} \mathrm{dose} \mathrm{group} \\
(n=6)\end{array}$ \\
\hline Phagocytic rate & $24.6 \pm 1.5$ & $22.1 \pm 1.8$ & $38.6 \pm 2.3$ & $36.9 \pm 1.9$ \\
Phagocytic index & $0.87 \pm 0.02$ & $0.77 \pm 0.15$ & $1.12 \pm 0.17$ & $0.99 \pm 0.24$ \\
\hline
\end{tabular}

TABLE 2: Effect of radix hedysari polysaccharide on the TNF- $\alpha$ signaling pathway in nude mice with glioma.

\begin{tabular}{lcccc}
\hline Group & $\begin{array}{c}\text { Normal nude mice } \\
(n=6)\end{array}$ & $\begin{array}{c}\text { Nude mice with glioma } \\
(n=6)\end{array}$ & $\begin{array}{c}150 \mu \mathrm{g} / \mathrm{ml} \text { dose group } \\
(n=6)\end{array}$ & $\begin{array}{c}400 \mu \mathrm{g} / \mathrm{ml} \mathrm{dose} \mathrm{group} \\
(n=6)\end{array}$ \\
\hline TNF- $\alpha(\mathrm{pg} / \mathrm{ml})$ & $16.65 \pm 1.56$ & $14.21 \pm 1.57$ & $21.69 \pm 2.73$ & $18.68 \pm 1.09$ \\
NF-KB p65 $(\mu \mathrm{g} / \mathrm{ml})$ & $27.69 \pm 3.45$ & $206.45 \pm 15.12$ & $69.16 \pm 7.46$ & $94.12 \pm 11.14$ \\
Caspase-3 $(\mu \mathrm{mol} / \mathrm{l})$ & $89.64 \pm 11.44$ & $80.69 \pm 9.46$ & $183.46 \pm 13.56$ & $126.67 \pm 10.82$ \\
\hline
\end{tabular}

resistant cells, and increasing tumor angiogenesis and metastasis [16]. However, it was reported that local and repeated injections of large amounts of human recombinant TNF- $\alpha$ can induce hemorrhagic tumor necrosis in homologous and xenograft tumors. By contrast, low-dose chronic TNF- $\alpha$ had angiogenic activity and promoted tumor progression [17]. In this study, the low dose of radix hedysari polysaccharide could significantly increase the levels of TNF- $\alpha$ and caspase- 3 in the serum of glioma nude mice and reduce the content of $\mathrm{NF}-\kappa \mathrm{B}$. The results suggest that radix hedysari polysaccharide can regulate the immune function of tumor mice, affecting the TNF- $\alpha$ signaling pathway to inhibit tumor development.

\section{Data Availability}

All the data is available with the handwritten notebook documented in our lab.

\section{Conflicts of Interest}

The authors declare that there are no conflicts of interest regarding the publication of this paper.

\section{Acknowledgments}

This work is supported by the Weifang Science and Technology Development Plan Project (Grant no. 2016YX023).

\section{References}

[1] L. M. DeAngelis, "Brain tumors," The New England Journal of Medicine, vol. 344, no. 2, pp. 114-123, 2001.

[2] X.-R. Feng, J.-X. Ding, R. Gref, and X.-S. Chen, "Poly( $\beta$-cyclodextrin)-mediated polylactide-cholesterol stereocomplex micelles for controlled drug delivery," Chinese Journal of Polymer Science, vol. 35, no. 6, pp. 693-699, 2017.

[3] S. Li, T. Zhang, W. Xu et al., "Sarcoma-targeting peptidedecorated polypeptide nanogel intracellularly delivers shikonin for upregulated osteosarcoma necroptosis and diminished pulmonary metastasis," Theranostics, vol. 8, no. 5, pp. 13611375, 2018.
[4] J. Chen, J. Ding, W. Xu et al., "Receptor and microenvironment dual-recognizable nanogel for targeted chemotherapy of highly metastatic malignancy," Nano Letters, vol. 17, no. 7, pp. 4526-4533, 2017.

[5] K. Zhao, D. Li, W. Xu et al., "Targeted hydroxyethyl starch prodrug for inhibiting the growth and metastasis of prostate cancer," Biomaterials, vol. 116, pp. 82-94, 2017.

[6] S. Zhong, D. F. Ji, Y. G. Li, T. B. Lin, Z. Q. Lv, and H. P. Chen, "Activation of P27kip1-cyclin D1/E-CDK2 pathway by polysaccharide from Phellinus linteus leads to S-phase arrest in HT-29 cells," Chemico-Biological Interactions, vol. 206, no. 2, pp. 222-229, 2013.

[7] P. Sitarek, E. Skała, M. Toma et al., "A preliminary study of apoptosis induction in glioma cells via alteration of the $\mathrm{Bax} / \mathrm{Bcl}-2-\mathrm{p} 53$ axis by transformed and non-transformed root extracts of Leonurus sibiricus L.," Tumor Biology, vol. 37, no. 7, pp. 8753-8764, 2016.

[8] D. Wei, Y. Wei, W. Cheng, and L. Zhang, "Sulfated modification, characterization and antitumor activities of Radix hedysari polysaccharide," International Journal of Biological Macromolecules, vol. 51, no. 4, pp. 471-476, 2012.

[9] J. Xie, D. T. Wu, W. Z. Li et al., "Effects of polysaccharides in Lycium barbarum berries from different regions of China on macrophages function and their correlation to the glycosidic linkages," Journal of Food Science, vol. 82, no. 10, pp. 24112420, 2017.

[10] L. Pinton, E. Masetto, M. Vettore, and S. Solito, "The immune suppressive microenvironment of human gliomas depends on the accumulation of bone marrow-derived macrophages in the center of the lesion," Journal for Immunotherapy of Cancer, vol. 7, no. 1, p. 58, 2019.

[11] D. Li, W. G. Xu, P. Q. Li et al., "Self-targeted polysaccharide prodrug suppresses orthotopic hepatoma," Molecular Pharmaceutics, vol. 13, no. 12, pp. 4231-4235, 2016.

[12] L. Di, J. X. Ding, X. L. Zhuang, L. Chen, and X. S. Chen, “Drug binding rate regulates the properties of polysaccharide prodrugs," Journal of Materials Chemistry B, vol. 4, no. 30, pp. 5167-5177, 2016.

[13] P. W. Szlosarek and F. R. Balkwill, "Tumour necrosis factor alpha:a potential target for the therapy of solid tumours," The Lancet Oncology, vol. 4, no. 9, pp. 565-573, 2003.

[14] Y. L. Cheng, C. L. He, J. X. Ding, C. S. Xiao, X. L. Zhuang, and X. S. Chen, "Thermosensitive hydrogels based on polypeptides 
for localized and sustained delivery of anticancer drugs," Biomaterials, vol. 34, no. 38, pp. 10338-10347, 2013.

[15] V. Haage, M. Semtner, R. O. Vidal, D. P. Hernandez, W. W. Pong, and Z. Chen, "Comprehensive gene expression metaanalysis identifies signature genes that distinguish microglia from peripheral monocytes/macrophages in health and glioma," Acta Neuropathologica Communications, vol. 7, no. 1, p. 20, 2019.

[16] A. Mantovani, P. Allavena, A. Sica, and F. Balkwill, "Cancerrelated inflammation," Nature, vol. 454, no. 7203, pp. 436444, 2008.

[17] A. Katsman, K. Umezawa, and B. Bonavida, "Chemosensitization and immunosensitization of resistant cancer cells to apoptosis and inhibition of metastasis by the specific NF- $\kappa \mathrm{B}$ inhibitor DHMEQ," Current Pharmaceutical Design, vol. 15, no. 7, pp. 792-808, 2009. 


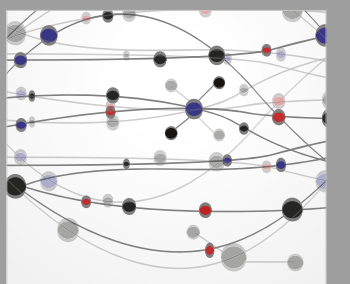

The Scientific World Journal
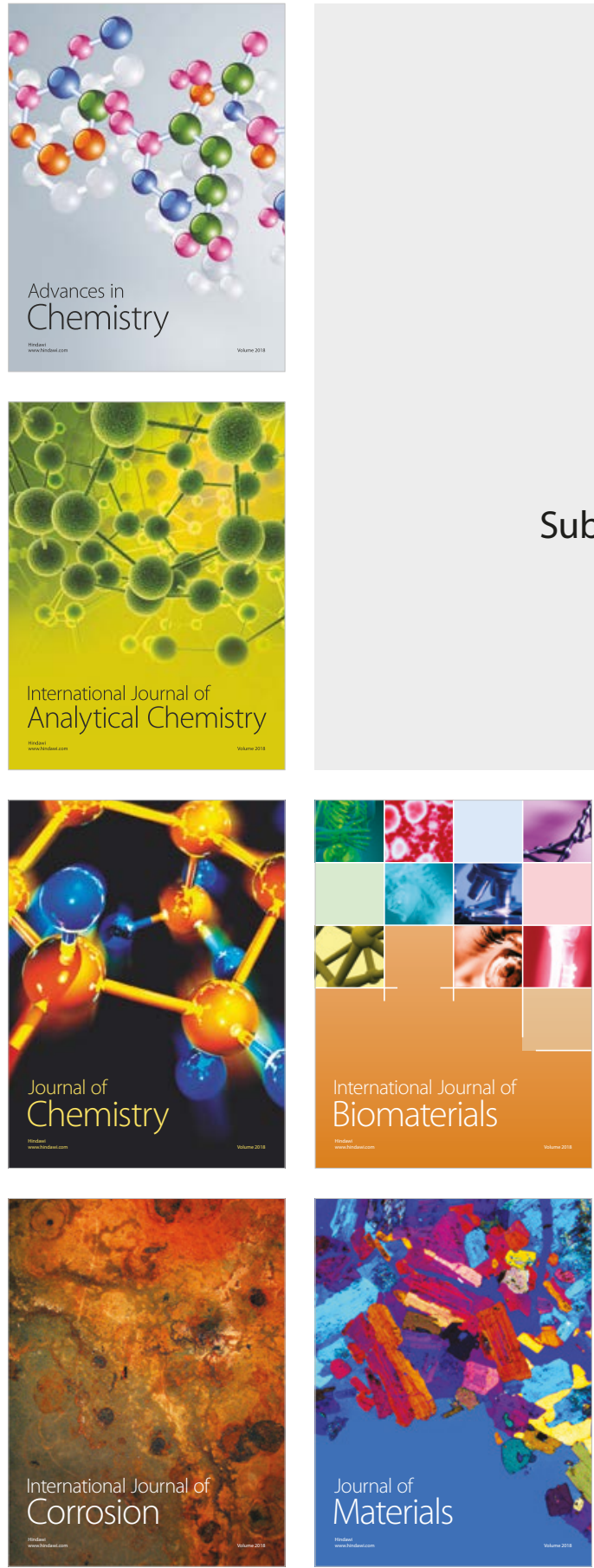

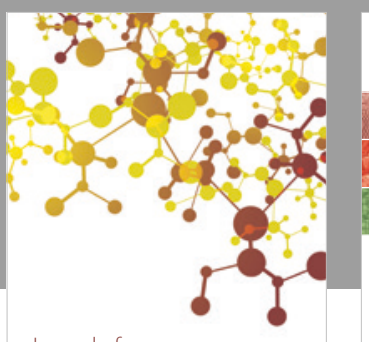

Journal of

Applied Chemistry
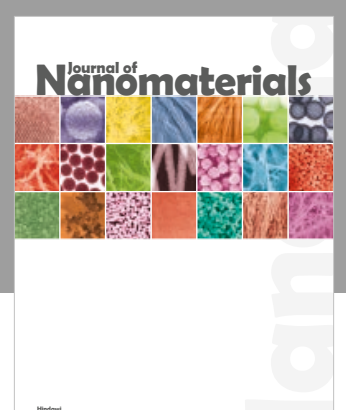

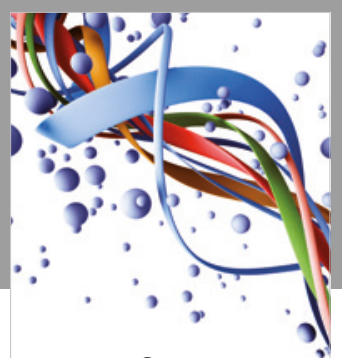

Scientifica

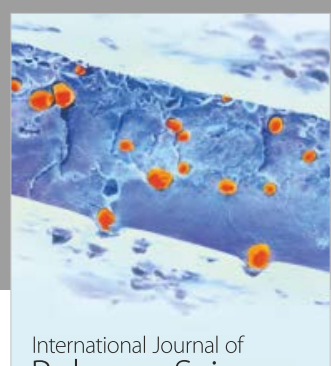

Polymer Science

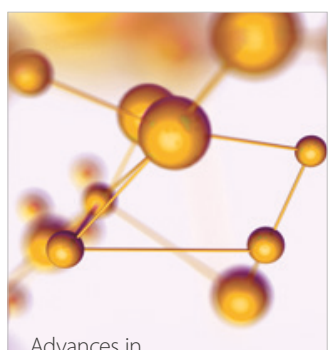

Physical Chemistry
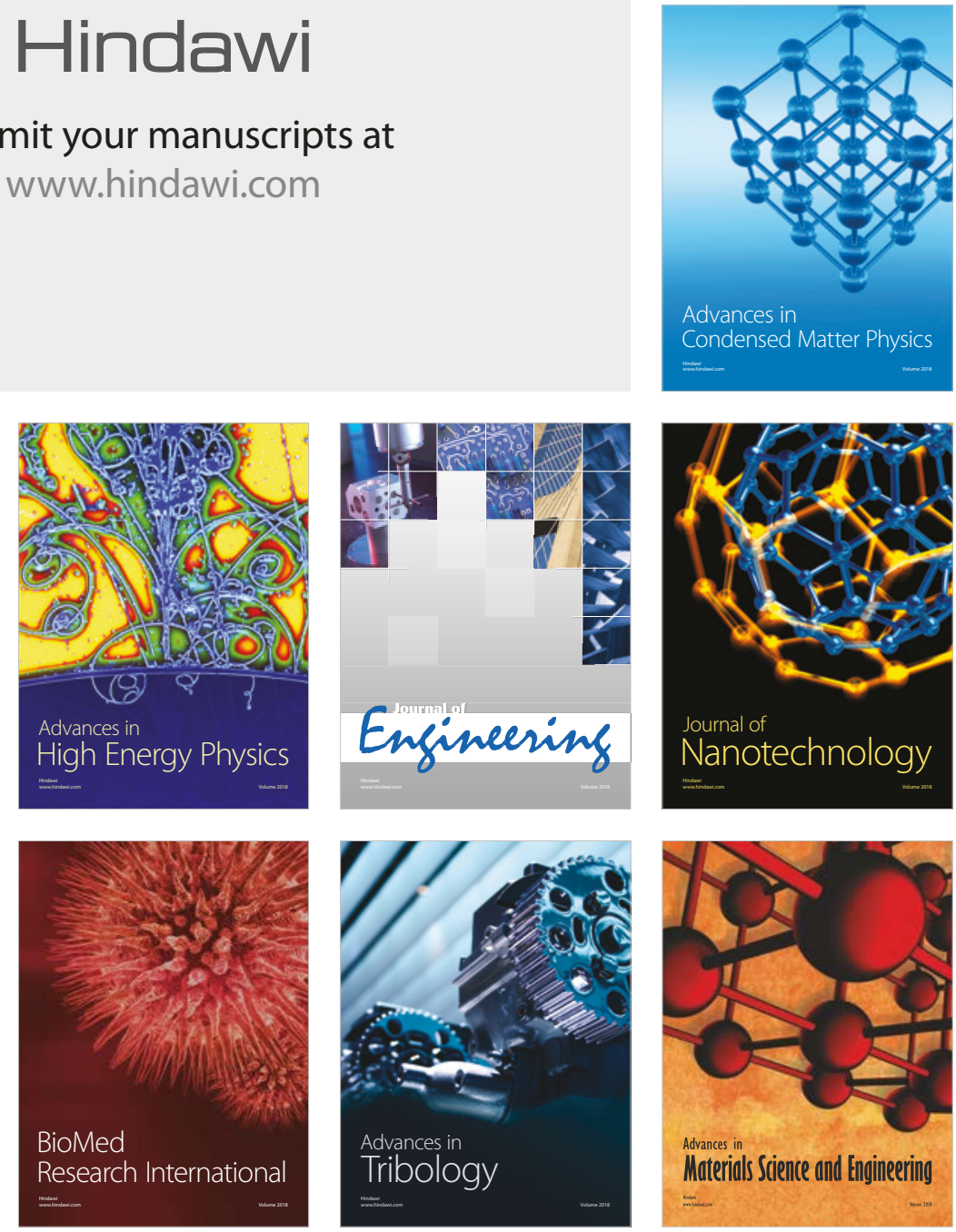